\title{
Three-component fluid dynamics for the description of energetic heavy-ion reactions
}

\author{
L. P. Csernai, I. Lovas, J. A. Maruhn, A. Rosenhauer, J. Zimányi, and W. Greiner \\ Institut für Theoretische Physik, Johann Wolfgang Goethe Universität, \\ D-6000 Frankfurt am Main 1, Germany \\ and Central Research Institute for Physics, 1525 Budapest, Hungary
}

(Received 28 December 1981)

\begin{abstract}
The nucleons taking part in heavy ion reaction are considered as a three-component fluid. The first and second components correspond to the nucleons of the target and the projectile, while the thermalized nucleons produced in the course of the collision belong to the third component. Making use of the Boltzmann equation, hydrodynamical equations are derived. An equation of state for anisotropic nuclear matter obtained from a field theoretical model in mean field approximation is applied in a one dimensional version of the three-component fluid model. The speed of thermalization is analyzed and compared to the results of cascade and kinetic models.
\end{abstract}

\section{[NUCLEAR REACTIONS Relativistic heavy-ion reactions, hydro- dynamic description.}

\section{INTRODUCTION}

In recent years the hydrodynamical model was applied successfully for the description of energetic heavy ion collisions. Owing to the assumption of immediate local equilibration, however, the collective flow arising from the high local pressure is somewhat overestimated in the model, while the conversion of kinetic energy into thermal excitations is inhibited, and hence insufficient entropy is produced. ${ }^{1}$ This entropy deficiency can be remedied to some extent by including viscous processes, ${ }^{2,3}$ but in the initial phase the deviations from local equilibrium are not small as it is assumed in viscous hydrodynamics. It was already pointed out $^{4-7}$ that the assumption of immediate local thermal equilibration may cause these deficiencies in the one-fluid hydrodynamical model. The first attempt to introduce a two fluid hydrodynamical model was made by Nix and his colleagues. ${ }^{8}$ This modification improved the agreement of the results with experimental ones at high energies. However, the two fluids maintained their identity during the first part of the collision process, leaving no room for real thermalization. The development of such a thermalized matter is important unless the nucleon-nucleon cross sections are extremely forward peaked (above $5 \mathrm{GeV} /$ nucleon), and therefore the scattered particles cannot lose their distinguishing longitudinal momenta in a few collisions. In fact, collective scattering effects like critical fluctuations may even at higher energies favor thermalization. ${ }^{9}$

In the hadron chemistry model ${ }^{10}$ the development of this third thermal component was taken into account, but collective effects like collective flow evolving from the interaction and pressure were neglected in the first nonthermalized stage of the collision. In another similar approach, ${ }^{7}$ the thermalization and shock front formation was analyzed in a one-dimensional many fluid model.

The process of thermal equilibration was studied by McLerran, ${ }^{11}$ by Cugnon et al. ${ }^{6}$ in a cascade approach, and by Randrup, ${ }^{12}$ Pirner and Schürmann, ${ }^{13}$ and Danielewicz ${ }^{14}$ in spatially homogeneous kinetic models, where the initial momentum distribution consisted of two identical displaced Fermi spheres. In the latter two calculations the time development of the whole momentum distribution was determined by solving the UehlingUhlenbeck transport equations, and rapid thermalization $(t \simeq 4-8 \mathrm{fm} / \mathrm{c}$ ) was found especially at lower beam energies. At higher energies the peaks around the initial position of target and projectile momenta maintained their position longer, and their amplitude was slowly decreasing while a thermal background component was evolving.

In the above mentioned kinetic models ${ }^{12-14}$ the spatial distribution was not considered. In principle, the cascade models ${ }^{6,15,16}$ should be a reasonable 
framework to describe the nucleon distributions. However, the neglections (e.g., long range interactions, triple or multiple collisions, assumed minimum of the nucleon free path) in the presently existing cascade models, inhibit the approach towards local equilibrium. Consequently, the collective processes are underestimated in these models.

A sophisticated multiple collision model was developed recently by Malfliet $^{17}$ where the Boltzmann transport equation is solved both in space and momentum variables. The components reaching different levels of thermalization were treated separately. However, this model suffers also from the problem of the kinetic theories that multiple collisions and long range interactions cannot be considered and so the collective effects are underestimated.

In the present work we try to incorporate both equilibrium and nonequilibrium aspects into a unified model; (i) the development of a thermalized matter component produced by the collisions, and (ii) the collective hydrodynamical motion of the thermalized and nonthermalized matter components.

We start from kinetic theory, but derive an approach which can be generalized to include physical properties of the real nuclear matter instead of those of an ideal gas. Hence, we will not be limited to the assumptions of the Boltzmann equation and dense systems may also be studied. In Sec. II A the two- and more-fluid hydrodynamical approaches are discussed and their connection to the transport theory is shown. From the possible approaches a simple version is selected in Sec. II B. It describes locally the gradual thermalization of the projectile and target nucleons. For this purpose at least a three-fluid hydrodynamical approach is necessary. In Sec. III the source terms of the three-fluid model are derived for an ideal nucleon gas, while in Sec. IV the source terms obtained in a relativistic mean field theory are discussed and extended to nonzero temperatures, and viscous and heat conductive processes. In Sec. V the results of a one dimensional test calculation and their consequences are presented.

\section{THE MULTICOMPONENT FLUID DYNAMICS}

\section{A. The equations of a multicomponent nonthermalized fluid}

The simplest theory which describes the above mentioned processes is the Boltzmann kinetic trans- port theory. Our model is based on this theory, but later we may include other ingredients (quantum and relativistic effects, long range interactions, etc.) as it is usually done in the derivation of the Euler and Navier-Stokes equations.

Let us suppose that the collision of two heavy ions at time $t$ can be characterized by the nucleon distribution function in the $\mu$-space: $f(\kappa, \sigma, t)$, normalized to the density

$$
n(\kappa, t)=\int d^{3} v f(\kappa, \bullet, t) .
$$

The time development of this distribution function is determined by the Boltzmann transport equation (external and long range forces are neglected)

$$
\left[\partial_{t}+(v \cdot \nabla)\right] f(\kappa, v, t)=C(\kappa, v, t) .
$$

Wherever the operator $\partial_{t}$ or $\nabla$ appears it acts on all quantities on the right in the same term. The collision term $C(\kappa, \omega, t)$ is defined as follows:

$$
\begin{aligned}
C=\frac{1}{2} \int & d^{3} v_{1} d^{3} v_{1}^{\prime} d^{3} v^{\prime} \\
\times & {\left[f\left(\kappa, v^{\prime}, t\right) f\left(\kappa, v_{1}^{\prime}, t\right) w\left(v^{\prime}, v_{1}^{\prime} \mid v_{,}\right)\right.} \\
& \left.\quad-f\left(\kappa, v_{1}\right) f\left(\kappa, v_{1}, t\right) w\left(v_{1} \mid v_{1}^{\prime}, v_{1}^{\prime}\right)\right] .
\end{aligned}
$$

Here the transition rate of the nucleon-nucleon elastic collisions is denoted by $w\left(v_{v}, v_{1} \mid v^{\prime}, v_{1}^{\prime}\right)$. If we multiply Eq. (1) with a quantity $\chi(0)$ which is conserved in the microscopic collisions (mass, momentum, and kinetic energy), and calculate the local expectation value of the equation by integrating over $d^{3} v$, the right hand side (collision integral) of the equation vanishes for any distribution function $f(x, \omega, t)$. The equations obtained this way will have a simple structure. Setting the $\chi(\sigma)$ equal to $m$, $m_{\bullet}$, and $m|\omega-u|^{2} / 2$, where

$$
u(\kappa, t)=\langle\omega\rangle=1 / n \int d^{3} v \sigma f(\kappa, v, t)
$$

is the average flow velocity, we get a set of equations equivalent to the hydrodynamical ones:

$$
\begin{aligned}
& {\left[\partial_{t}+(\nabla \cdot u)\right] \rho(\kappa, t)=0,} \\
& {\left[\partial_{t}+(\nabla \cdot u)\right]\left(\rho(\kappa, t)_{\varkappa}\right)=-(\nabla \cdot P),} \\
& {\left[\partial_{t}+(\nabla \cdot \mu)\right] \epsilon(\kappa, t)=-(\nabla \cdot g)-(\Lambda: P),}
\end{aligned}
$$

where $\rho(\kappa, t)$ is the total mass density, $\rho(\kappa, t)=m n$, $\epsilon(\kappa, t)$ is the energy density without the rest mass,

$$
\epsilon(\kappa, t)=-m\left\langle|\omega-u|^{2}\right\rangle / 2,
$$

$g(\kappa, t)$ is the heat flux (multiplied by the rest mass), 


$$
g(\mu, t)=\rho\left\langle(\sigma-u)|\sigma-u|^{2}\right\rangle / 2,
$$

$P^{i j}(\mu, t)$ is the pressure tensor (superscripts are spatial indices),

$$
\begin{aligned}
P^{i j}(\kappa, t) & =\rho\left\langle\left(v^{i}-u^{i}\right)\left(v^{j}-u^{j}\right)\right\rangle \\
& =\rho\langle(v-u) \circ(v-u)\rangle,
\end{aligned}
$$

and in the last equation the tensor $\Lambda^{i j}(\mu, t)$ contains the derivatives of the collective flow velocity,

$$
\Lambda^{i j}(\kappa, t)=\left(\partial_{j} u^{i}+\partial_{i} u^{j}\right) / 2=[\nabla \circ u]_{\mathrm{sym}} .
$$

$(\nabla \cdot P)$ is a vector whose $i$ th component is $\Sigma_{j} \partial_{j} p^{i j}$ and

$$
(\Lambda: P)=\Sigma_{i j} \Lambda^{i j} P^{i j} .
$$

These equations are valid for any distribution $f(x, w, t)$. If we suppose that the momentum distribution is a locally thermalized (Maxwell-
Boltzmann) distribution $f_{0}$, then Eqs. (3) reduce to the Euler equations of the hydrodynamics. If the distribution $f$ is close to a local equilibrium distribution the quantities $P^{i j}$ and $q$ can be evaluated and the Navier-Stokes equations are obtained.

When the system is far away from the thermal equilibrium, in some cases, as it will be shown, it is useful to decompose the distribution function of the indistinguishable particles in the following way:

$$
f(\kappa, \omega, t)=\Sigma_{i=1, N} f_{i}(\kappa, v, t) .
$$

The type and number of components $f_{i}$ depend on the specific physical problem to be discussed. On the basis of the Boltzmann equation, a coupled set of equations may be introduced as

$$
\left[\partial_{t}+(\omega \cdot \nabla)\right] f_{i}(\kappa, v, t)=C_{i}(\kappa, v, t)
$$

where the collision terms $C_{i}$ are defined by

$$
C_{i}=\frac{1}{2} \Sigma_{j k} \int d^{3} v_{1} d^{3} v_{1}^{\prime} d^{3} v^{\prime}\left[f_{j}\left(\mu, v^{\prime}, t\right) f_{k}\left(\mu, v_{1}^{\prime}, t\right) w_{i}\left(v^{\prime}, v_{1}^{\prime} \mid v_{,}\right)-\delta_{i j} f_{j}\left(\mu, v^{\prime}, t\right) f_{k}\left(\mu, v_{1}, t\right) w\left(v_{,}, v_{1} \mid v^{\prime}, v_{1}^{\prime}\right)\right] .
$$

Here the partial transition rates $w_{i}\left(v^{\prime}, v_{1}^{\prime} \mid v_{,}, v_{1}\right)$ give the transition probability to the final state, characterized by the velocities $v$ and $v_{1}$, under the condition that the nucleon of velocity o belongs to the component $f_{i}(\kappa, v, t)$ after the collision. Since the particles are indistinguishable, the transition probability is independent of the initial components. If the sum of the partial transition rates is equal to the total one, i.e.,

$$
\Sigma_{i} w_{i}\left(v^{\prime}, v_{1}^{\prime} \mid v_{,} v_{1}\right)=w\left(v^{\prime}, v_{1}^{\prime} \mid v_{,} v_{1}\right),
$$

then the sum of the solutions of the coupled set of equations (5) satisfies the original Boltzmann equation.

If one wants to solve the Boltzmann equation in its full complexity then, of course, it is better to use the single equation instead of the coupled set of equations. If we want, however, to solve the problem only approximately, e.g., by the method of moments, then the decomposition of the distribution function may be useful because in this way the lowest order moments of the distribution function $f_{i}$, namely the density, the flow velocity, and the energy density, may carry information about the system which otherwise is contained only in the higher order moments of the distribution function $f$. On the other hand, by the decomposition of the distribution function $f$ we can introduce physically plausible assumptions which cannot be formulated at all if we use the original Boltzmann equation.

The partial transition rates are uniquely defined only if the distribution functions $f_{i}$ do not overlap with each other. In the heavy ion collisions, however, this is not the case, and therefore, the decomposition of the total transition rate is arbitrary. It should be noted, however, that the decomposition of the distribution function $f$ into components $f_{i}$ is also completely arbitrary. The decomposition is defined by the prescription of the partial transition rates, by the initial conditions, and by the assumed functional form of the component distributions $f_{i}$. This mathematical freedom gives us the possibility of introducing the appropriate physical assumptions, reflecting the specific features of the physical system studied.

Now we derive hydrodynamical equations for the multicomponent fluid. Similar to the way Eq. (3) is obtained $^{18}$ but starting from Eq. (5) we end up at the following set of equations:

$$
\begin{aligned}
{\left[\partial_{t}+\left(\nabla \cdot u_{i}\right)\right] n_{i}=} & \int C_{i} d^{3} v, \\
{\left[\partial_{t}+\left(\nabla \cdot u_{i}\right)\right] n_{i} u_{i} } & =-\left(\nabla \cdot P_{i}\right) / m+\int C_{i} \bullet d^{3} v, \\
{\left[\partial_{t}+\left(\nabla \cdot \mu_{i}\right)\right] \epsilon_{i}=} & -\left(\nabla \cdot g_{i}\right)-\left(\Lambda_{i}: P_{i}\right) \\
& +\int C_{i} m\left(\mho-u_{i}\right)^{2} / 2 d^{3} v,
\end{aligned}
$$


where the notations are defined as follows: the partial particle number density

$$
n_{i}(\kappa, t)=\int f_{i}(\kappa, u, t) d^{3} v
$$

the flow velocity

$$
u_{i}=n_{i}{ }^{-1} \int f_{i}(\kappa, v, t)_{v} d^{3} v \equiv\langle v\rangle_{i},
$$

the energy density

$$
\epsilon_{i}(\kappa, t)=m\left\langle\left(\sigma-u_{i}\right)^{2}\right\rangle_{i} / 2,
$$

the pressure tensor

$$
P_{i}(\kappa, t)=m n_{i}\left\langle\left(\sigma-u_{i}\right) \circ\left(\sigma-u_{i}\right)\right\rangle_{i},
$$

the heat flux

$$
g_{i}(\kappa, t)=m n_{i}\left\langle\left(\omega-\mu_{i}\right)\left|\omega-u_{i}\right|^{2}\right\rangle_{i} / 2,
$$

and finally the derivative tensor of the flow velocity

$$
\Lambda_{i}(\kappa, t)=\left[\nabla \circ \mu_{i}(\kappa, t)\right]_{\mathrm{sym}} .
$$

In contrast to the case when the original Boltzmann equation is used, the collision integrals standing on the right hand side of Eqs. (8) do not vanish. These collision integrals are responsible for the mass, momentum, and energy transfer among the components. These equations can be considered as the generalization of the conventional hydrodynamics for a multicomponent fluid. The main field of the application of this theory is the analysis of nonequilibrium processes, where the characteristic time of local equilibration and that of the collective flow are of the same order of magnitude.

In order to avoid misunderstanding it is necessary to emphasize that the multicomponent fluid is essentially different from the mixture of different fluids where the particles are physically distinguishable (e.g., proton and neutron fluids). In this latter case the particle transport between different fluids is not possible, hence the hydrodynamical equations describing such a system are somewhat simpler. ${ }^{19}$ The multicomponent fluid dynamics seems to provide an appropriate tool to follow the process of the thermalization which is rather difficult in the framework of the conventional two-fluid dynamics. $^{8,19}$

A serious problem arises, however, in this separated multicomponent fluid dynamical approach when we want to generalize the source terms by including the long range interactions, ${ }^{20,21}$ viscous and heat conducting processes. In this case the source terms are not separable any more (see Sec. IV). Nevertheless, in Sec. II B we shall overcome this difficulty.

A clear and simple separation of the Boltzmann equation was introduced by Malfliet in the multiple collision model. ${ }^{17}$ The distribution function was separated into an infinite number of components. A given component $i$ contained the particles scattered $i$ times and so the transition probabilities $w_{i}$ could be evaluated in a straightforward way. However, to handle this infinite set of separated Boltzmann equations additional simplifying assumptions had to be applied and so the spatial and time variations of the system could be studied only in a limited way.

In order to analyze the structure of Eqs. (8) let us express the collision terms in the usual manner with the help of the effective cross section of the elastic scattering and the relative velocity ${ }^{18}$

$$
C_{i}=\int d^{3} v_{1} d \Omega \Sigma_{j k}\left|\mho-\mho_{1}\right|\left[f_{j}\left(\kappa, \iota^{\prime}, t\right) f_{k}\left(\kappa, \mho_{1}^{\prime}, t\right) \sigma_{i}(\Omega)-\delta_{i j} f_{j}(\kappa, \vartheta, t) f_{k}\left(\kappa, \mho_{1}, t\right) \sigma(\Omega)\right] .
$$

If we assume that in the collision integrals the product of the cross section and the relative velocity can be replaced by a mean value defined as

$$
\left\langle\sigma v_{\mathrm{rel}}\right\rangle_{i k} \equiv \frac{\left[\int f_{i}(\kappa, \omega, t) f_{k}\left(\kappa, v_{1}, t\right) \sigma(\Omega)\left|\mho-v_{1}\right| d \Omega d^{3} v_{1} d^{3} v\right]}{\left[\int f_{i}(\kappa, \omega, t) f_{k}\left(\kappa, v_{1}, t\right) d^{3} v d^{3} v_{1}\right]}
$$

then the collision term can be written as

$$
C_{i} \simeq \Sigma_{j k}\left[\left\langle\sigma_{i} v_{\text {rel }}\right\rangle_{j k} \int d^{3} v_{1} f_{j}\left(\kappa, v^{\prime}, t\right) f_{k}\left(\kappa, v_{1}^{\prime}, t\right)-\delta_{i j}\left\langle\sigma v_{\text {rel }}\right\rangle_{j k} \int d^{3} v_{1} f_{j}(\kappa, \omega, t) f_{k}\left(\kappa, \iota_{1}, t\right)\right] .
$$

This approximation has the advantage that Eqs. (8) can be written in a simple and self-explanatory separated form 


$$
\begin{aligned}
& {\left[\partial_{t}+\left(\nabla \cdot u_{i}\right)\right] n_{i}=\Sigma_{j k}\left[\left\langle\sigma_{i} v_{\mathrm{rel}}\right\rangle_{j k} n_{j} n_{k}-\delta_{i j}\left\langle\sigma v_{\mathrm{rel}}\right\rangle_{j k} n_{j} n_{k}\right],} \\
& {\left[\partial_{t}+\left(\nabla \cdot u_{i}\right)\right] n_{i} u_{i}=\Sigma_{j k}\left[\left\langle\sigma_{i} v_{\mathrm{rel}}\right\rangle_{j k} n_{j} n_{k}\left(u_{j}+u_{k}\right) / 2-\delta_{i j}\left\langle\sigma v_{\mathrm{rel}}\right\rangle_{j k} n_{j} n_{k} u_{i}\right]-\left(\nabla \cdot P_{i}\right) / m,} \\
& {\left[\partial_{t}+\left(\nabla \cdot u_{i}\right)\right] \epsilon_{i}=\Sigma_{j k}\left[\left\langle\sigma_{i} v_{\mathrm{rel}}\right\rangle_{j k}\left(\epsilon_{j} n_{k}+\epsilon_{k} n_{j}\right) / 2-\delta_{i j}\left\langle\sigma v_{\mathrm{rel}}\right\rangle_{j k} n_{j} \epsilon_{j}\right]-\left(\Lambda: P_{i}\right)-\left(\nabla \cdot g_{i}\right) .}
\end{aligned}
$$

In this approximation, as it is seen from the second and third equation of (12), the removal of a particle from a given component is associated with the removal of the average value of the momentum and energy, thus keeping the momentum/particle and energy/particle ratio of this component unchanged. However, the momentum/particle and energy/ particle ratio of the component into which the particle is scattered will be changed.

\section{B. Three-component fluid dynamics for heavy ion reactions}

Let us assume that the distribution can be physically decomposed into three components and all three components can be described by an equilibrated distribution (Fig. 1). The parameters of the distributions are then $n_{i}, u_{i}$, and $T_{i}$ :

$$
f(\kappa, v, t)=\Sigma_{i=1,3} f_{i}(\kappa, v, t) .
$$

It follows from Eq. (13) by definition that

$$
n=\Sigma_{i=1,3} n_{i} \text { and } n_{\mu}=\Sigma_{i=1,3} n_{i} u_{i},
$$

where $n_{i}$ and $\mu_{i}$ are the partial density and mean flow velocity of component $i$, respectively. When we want to apply Eqs. (8) or Eqs. (12) to determine the time dependence of these parameters a serious problem arises.

We know that summing up over $i$ in Eqs. (8) we must obtain the conventional equations of hydrodynamics, Eqs. (3). But this is true only if the quantities $C_{i}, P_{i}$, and $q_{i}$ are calculated by means of distribution functions $f_{i}$ satisfying the Boltzmann equations (5). However, the distributions $f_{i}$ are not known and so we are forced to introduce some assumptions for $f_{i}$ when we evaluate the quantities $C_{i}, P_{i}$, and $g_{i}$. Therefore, having summed up Eqs. (8) obtained in this way the recovery of the hydrodynamical equations (3) is not ensured, in other words, the general conservation laws might be violated.

The only possibility of avoiding this problem is to explicitly require Eq. (3) to be satisfied. In order to get a nonoverdetermined set of equations we can then omit from Eqs. (8) the set of equations describing one of the three components:

$$
\begin{aligned}
& {\left[\partial_{t}+(\nabla \cdot u)\right] \rho(\kappa, t)=0,} \\
& {\left[\partial_{t}+(\nabla \cdot u)\right](\rho(\kappa, t) u)=-(\nabla \cdot P),} \\
& {\left[\partial_{t}+(\nabla \cdot u)\right] \epsilon(\kappa, t)=-(\nabla \cdot g)-(\Lambda: P),} \\
& {\left[\partial_{t}+\left(\nabla \cdot u_{i}\right)\right] n_{i}=\int C_{i} d^{3} v,} \\
& {\left[\partial_{t}+\left(\nabla \cdot u_{i}\right)\right] n_{i} u_{i}=-\left(\nabla \cdot P_{i}\right) / m+\int C_{i} d^{3} v,} \\
& {\left[\partial_{t}+\left(\nabla \cdot u_{i}\right)\right] \epsilon_{i}=-\left(\nabla \cdot g_{i}\right)-\left(\Lambda_{i}: P_{i}\right)} \\
& \quad+\int C_{i} m\left(\mho-u_{i}\right)^{2} / 2 d^{3} v, \\
& \quad(i=1,2)
\end{aligned}
$$

On the basis of the physical process we want to discuss, we can use the following simplifying assumptions: Each nucleon-nucleon collision between par-
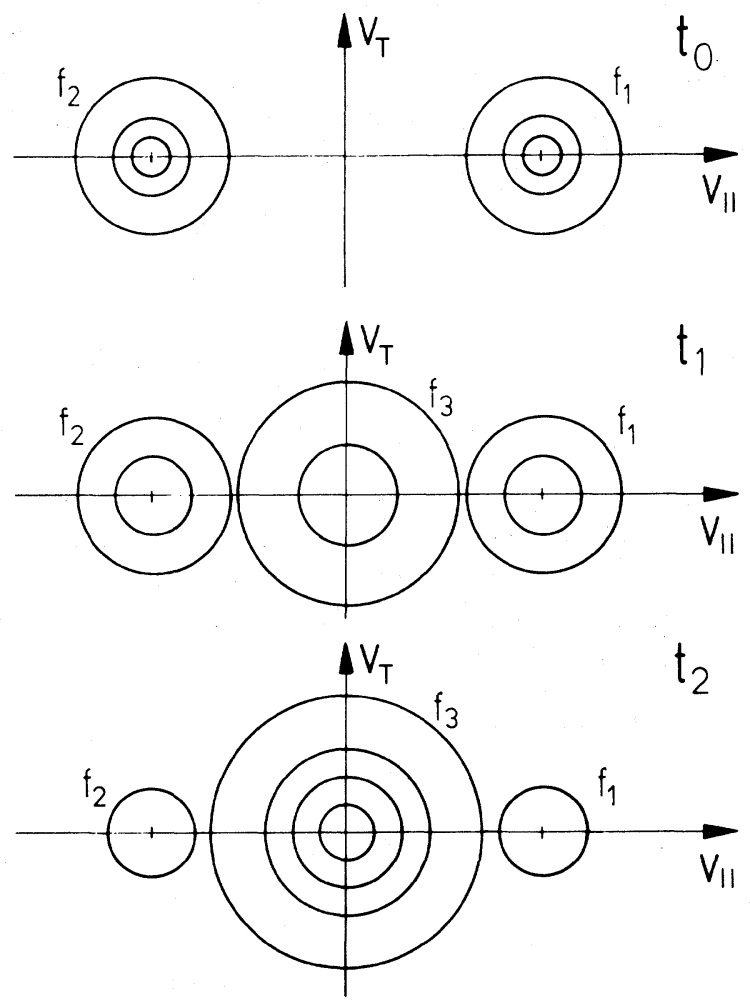

FIG. 1. Schematic plot of the time evolution of the velocity distribution in the three-component fluid approximation. The density is proportional to the density of the contour lines. 
ticles belonging to different components, $(i j)$ $=(1,2),(1,3)$, and $(2,3)$, populates the third component describing the thermalized particles, and so we can assume that the projectile and target components, $i=1,2$, move with constant velocities, $u_{1}=$ const, $u_{2}=$ const, and their momentum distribution is not changing during the reaction $T_{1}=$ const, $T_{2}=$ const. These assumptions are the local equivalents of the hadron chemistry model of Montvay and Zimányi ${ }^{10}$ and are motivated by the study of the time development of the nucleon momentum distribution ${ }^{12}$ in heavy ion reactions. It was found in a model calculation ${ }^{22}$ that the momentum distribution of the once scattered nucleon generation can be approximated by a thermal distribution if the projectile energy is relatively small $\left(E_{p}<0.6 \mathrm{GeV} /\right.$ nucleon), and so the initial target and projectile momentum distributions are not disjoint spheres in momentum space. At higher energies the momentum distribution of the collided particles is elongated in the beam direction so that the anisotropy of this component distribution has to be taken into account.

According to the assumptions above, a collision between different components does not populate $f_{1}$ and $f_{2}$. The flow velocity and the temperature are fixed for the components 1,2 and the continuity equation [first Eq. in (8)] is simpler because only the loss term is present in the collision integral:

$$
\begin{aligned}
& {\left[\partial_{t}+\left(u_{i} \cdot \nabla\right)\right] n_{i}=-\Sigma_{k \neq i} \int d^{3} v_{1} d^{3} v_{1}^{\prime} d^{3} v^{\prime}} \\
& \times f_{i}(\kappa, \omega, t) f_{k}\left(\kappa, \omega_{1}, t\right) \\
& \times w\left(v_{1} v_{1} \mid v^{\prime}, v_{1}^{\prime}\right) / 2 \\
& =-\Sigma_{k \neq i} Z^{i k} \quad(i=1,2) \text {. }
\end{aligned}
$$

Completing the hydrodynamical equation set for the whole matter with the continuity equation for the target and projectile components from the separated approach (8) we thus obtain a fully determined set of equations:

$$
\begin{aligned}
& {\left[\partial_{t}+(\nabla \cdot u)\right] n=0, } \\
& {\left[\partial_{t}+(\nabla \cdot u)\right](\rho u)=-(\nabla \cdot P), } \\
& {\left[\partial_{t}+(\nabla \cdot u)\right] \epsilon=-(\nabla \cdot q)-(\Lambda: P), } \\
& {\left[\partial_{t}+(\nabla \cdot u)\right] n_{i}=-\left(\Delta u_{i} \cdot \nabla\right) n_{i}+n_{i}(\nabla \cdot u) } \\
&-\Sigma_{k \neq i} Z_{i k}, \quad(i=1,2),
\end{aligned}
$$

where $\Delta \mu_{i}=u_{i}-u$ and $\rho=n m$. To solve this system of equations we need the pressure tensor $P^{k l}$, the collision numbers $Z_{12}, Z_{13}, Z_{23}$, the heat conduction vector $g$ in the nonequilibrium matter, and the relation between the energy density $\epsilon$ and the other parameters of the model $\left(n_{i}, T_{i}, \Delta u_{i}, i=1,3\right)$.

Equations (17) are general conservation laws, therefore more realistic source terms ${ }^{20}$ can also be applied. The number of differential equations to be solved in the one- and two-fluid model and in the three-component fluid model is 5,10 , and 15 , respectively, while in the approach described above this number is 7 .

This approach is essentially different from the two-fluid model elaborated in Los Alamos. ${ }^{8}$ Here the thermalized matter component coexists with the target and projectile components, and the particles are allowed to be exchanged among the components.

\section{SOURCE TERMS IN THE CASE OF AN IDEAL BOLTZMANN GAS MIXTURE}

Equations (17) contain the source terms $q, P^{i j}$, and $Z_{k l}$. The evaluation of the first two quantities is relatively simple if all of the components $f_{i}$ are Maxwell-Boltzmann distributions:

$$
\begin{aligned}
f_{i}=f_{i}^{0}(\kappa, \bullet, t)= & n_{i}\left(m / 2 \pi T_{i}\right)^{3 / 2} \\
& \times \exp \left[-m\left(\bullet-u_{i}\right)^{2} / 2 T_{i}\right] .
\end{aligned}
$$

From the definition of the energy density:

$$
\begin{aligned}
\epsilon & =n m\left\langle|v-u|^{2}\right\rangle / 2 \\
& =0.5 m \Sigma_{i=1,3} \int d^{3} v|v-u|^{2} f_{i}^{0}(\kappa, v, t) .
\end{aligned}
$$

Introducing local coordinate systems in each term, $\mathscr{U}_{i}=v-u_{i}$, the square of the velocity difference can be written as

$$
\left|\mathscr{U}_{i}+\Delta u_{i}\right|^{2}=U_{i}^{2}+\Delta u_{i}^{2}+2\left(\mathscr{U}_{i} \cdot \Delta u_{i}\right),
$$

where $\Delta u_{i}=u_{i}-u$. Since $f_{i}^{0}$ is spherically symmetric in coordinates $\mathscr{U}_{i}$ the last term does not contribute to the integral and so we obtain

$$
\begin{aligned}
\epsilon & =\Sigma_{i=1,3}\left(\epsilon_{T i}+m \Delta u_{i}{ }^{2} n_{i} / 2\right) \\
& =\Sigma_{i=1,3} n_{i}\left(3 T_{i}+m \Delta u_{i}{ }^{2}\right) / 2 .
\end{aligned}
$$

Here, according to the ideal gas assumption, the contribution of interactions to the energy density is neglected. In Sec. IV it will be pointed out how this and the following source terms may be modified in the case of an interacting Fermi system.

For the calculation of the heat conduction we use 
the same velocity coordinates

$g=0.5 m \Sigma_{i=1,3} \int d^{3} U_{i}\left(\mathscr{U}_{i}+\Delta u_{i}\right)\left|\mathscr{U}_{i}+\Delta u_{i}\right|^{2} f_{i}^{0}$.

In the integral three nonvanishing terms remain:

$$
\begin{aligned}
g=0.5 m \Sigma_{i=1,3} \int d^{3} U_{i}[ & \Delta u_{i} \Delta u_{i}{ }^{2}+\Delta u_{i} U_{i}^{2} \\
& \left.+2 \mathscr{U}_{i}\left(\mathscr{U}_{i} \cdot \Delta u_{i}\right)\right] f_{i}^{0},
\end{aligned}
$$

and so the heat conduction is

$$
\begin{aligned}
g & =\Sigma_{i=1,3} \Delta u_{i}\left(n_{i} m \Delta u_{i}^{2} / 2+\frac{5}{3} \epsilon_{i 0}\right) \\
& =\Sigma_{i=1,3} g_{i} .
\end{aligned}
$$

The definition of the pressure tensor $P^{i k}$ is

$$
P^{j k}=m \Sigma_{i=1,3} \int d^{3} v\left(v^{j}-u^{j}\right)\left(v^{k}-u^{k}\right) f_{i}^{0},
$$

and similar to the previous calculations we obtain:

$$
\begin{aligned}
P^{j k} & =\Sigma_{i=1,3}\left[p_{i} \delta^{j k}+n_{i} m \Delta u_{i}^{j} \Delta u_{i}^{k}\right] \\
& =\Sigma_{i=1,3} P_{i}^{j k},
\end{aligned}
$$

where $p_{i}=\frac{2}{3} \epsilon_{T i}$.

The evaluation of the collision integral $Z_{i k}$ leads to integrals not expressible in analytic form. Some limiting cases, however, can be written down. If the parameters of the two components $i, k$ are identical the collision integral is given by ${ }^{18}$ :

$$
Z_{i k}=\sigma_{\text {tot }} n_{i} n_{k} 4 \sqrt{T / m \pi} .
$$

In the extreme limiting case when the thermal velocities are negligible compared to the difference between the mean velocities, $\sqrt{2 T / m}<D$, $\left(\mathscr{D}=u_{i}-u_{k}\right)$ one obtains

$$
Z_{i k}=\sigma_{\text {tot }} n_{i} n_{k} D
$$

If the two components have different temperatures but $D=0$, the collision integral is

$$
\begin{aligned}
Z_{i k}= & \sigma_{\text {tot }} n_{i} n_{k}(m / 2 \pi)^{3}\left(T_{i} T_{k}\right)^{-3 / 2} \\
& \times \int d^{3} V e^{-\gamma V^{2} 4 \pi} \\
& \times \int d v v^{3} e^{-\gamma v^{2} / 4} \operatorname{sh}(\delta v V) /(\delta v V),
\end{aligned}
$$

where

$$
\begin{aligned}
& \mathscr{V}=\left(v_{i}+v_{k}\right) / 2, \\
& v=v_{i}-v_{k}, \\
& \gamma=m\left(1 / T_{i}+1 / T_{k}\right) / 2,
\end{aligned}
$$

and

$$
\delta=m\left(1 / T_{i}-1 / T_{k}\right) / 2 .
$$

Let us expand the function $s h(x)$ up to second order (i.e., for small $\delta$ parameters) and perform the integrations. We obtain

$$
\begin{aligned}
Z_{i k} & =\sigma_{\text {tot }} n_{i} n_{k} 4 \sqrt{\left(T_{i} T_{k}\right)^{4} m \pi T^{7}}\left[1+(\Delta T / T)^{2} / 2\right] \\
& \simeq \sigma_{\text {tot }} n_{i} n_{k} 4 \sqrt{T / m \pi}\left[1+(\Delta T / T)^{2} / 2\right],
\end{aligned}
$$

where $T=\left(T_{i}+T_{k}\right) / 2$, and $\Delta T=\left(T_{i}-T_{k}\right)$. Similarly for small mean velocity difference $D<\sqrt{2 T / \pi m}$ one gets

$$
Z_{i k}=\sigma_{\mathrm{tot}} n_{i} n_{k} 4 \sqrt{T / m \pi}\left[1+D^{2} m / 3 T\right] .
$$

The simple expression $Z_{i k}(D)=c \sqrt{D^{2}+b}$, where $c=\sigma_{\text {tot }} n_{i} n_{k}$ and

$$
b=(16 T / m \pi)\left[1+(\Delta T / T)^{2} / 2\right]^{2},
$$

approximates relatively well the asymptotic behavior $(D \rightarrow 0, D \rightarrow \infty, \Delta T \rightarrow 0)$ of the collision integral in the case of two Boltzmann gases.

Summarizing the results in the case of a distribution $f$ with three Maxwell-Boltzmann components, Eq. (13), the source terms [right hand sides of Eqs. (17)] contain the quantities

$$
P^{k j}=\Sigma_{i}\left[p_{i} \delta^{k j}+n_{i} m\left(\Delta u_{i}^{k} \Delta u_{i}^{j}\right)\right],
$$

where

$$
\begin{aligned}
& p_{i}=m / 3 \int U_{i}^{2} f_{i} d^{3} U_{i}=n_{i} T_{i}, \\
& \mathscr{g}=\Sigma_{i=1,3} \Delta u_{i}\left(n_{i} m \Delta u_{i}^{2} / 2+\frac{5}{3} \epsilon_{T i}\right), \\
& \epsilon=\Sigma_{i=1,3}\left(n_{i} m \Delta u_{i}^{2} / 2+\epsilon_{T i}\right),
\end{aligned}
$$

with $u_{i}$ being the average velocity of the partial distribution $f_{i}(\mu, v, t), \mathscr{U}_{i}$ is the relative velocity of particles in $f_{i}$ with respect to $u_{i}$, i.e., $\mathscr{U}_{i}=v_{i}-u_{i}$, and $\Delta \varkappa_{i}$ is the difference of the average velocities in the rest system of the whole matter, $\Delta u_{i}=u_{i}-u$. Since we assumed Maxwell-Boltzmann component distributions, the partial energy density of one component is $\epsilon_{T i}=3 n_{i} T_{i} / 2$. The total energy density contains these partial energy densities and the kinetic energy arising from the partial flow velocities $\Delta u_{i}$ in the rest frame of the whole matter.

\section{THE PRESSURE TENSOR AND THE ENERGY DENSITY}

\section{A. Inclusion of realistic nuclear matter properties}

In Ref. 20 the equation of state for anisotropic, cold nuclear matter is studied in the framework of Walecka's mean field theory. The energy density 
and the pressures are calculated for two simple cases which may be relevant in the description of heavy ion collisions: (i) the Fermi surface consists of two, nonoverlapping, Lorentz elongated spheres, and (ii) the Fermi surface is a reflection and axially symmetric ellipsoid.

It should be noted that in both cases studied in Ref. 20 the nondiagonal elements of the stress tensor vanish because of the axial and reflection symmetries of the system. Furthermore, it is worthwhile to emphasize that the pressure in the $z$ direction is considerably greater than in the perpendicular directions, similar to the simple Boltzmann gas approximation. Note also that the pressure components in the perpendicular directions are also affected by the anisotropy.

For the application in a three component fluid dynamical model, the following approximate expressions are satisfactory, and reflect the basic properties of the source terms obtained in the above $\operatorname{model}^{20}$ :

$$
\begin{aligned}
& \epsilon= \epsilon_{c}(n)+\epsilon_{T}\left(n_{i}, T_{i}\right)+\epsilon_{\mathrm{int}}\left(n_{i}, T_{i}, \Delta \mu_{i}\right) \\
&=\epsilon_{c}(n)+\epsilon_{\mathrm{res}}, \\
& P^{j k}=\left[p_{c}(n)+p_{T}\left(n_{i}, T_{i}\right)\right] \delta^{j k} \\
& \quad+P_{\mathrm{int}}^{j k}\left(n_{i}, T_{i}, \Delta \mu_{i}\right)+P_{\mathrm{visc}}^{j k} \\
&=p_{c} \delta^{j k}+P_{\mathrm{res}}^{j k},
\end{aligned}
$$

where $p_{c}(n)=n \partial \epsilon_{c}(n) / \partial n-\epsilon_{c}(n)$. (This relation can always be used if there is no phase transition in the matter.) The usual relations of equilibrium thermodynamics connecting the whole pressure tensor and energy density are not valid in our case. In the present calculations the temperature dependence of functions $\epsilon_{T}$ and $p_{T}$ is approximated by the thermal energy and thermal pressure of the ideal gas as in Sec. III:

$$
\begin{aligned}
& \epsilon_{T}=\Sigma_{i} \epsilon_{T i}=\Sigma_{i} 3 n_{i} T_{i} / 2, \\
& p_{T}=\frac{2}{3} \epsilon_{T} .
\end{aligned}
$$

According to the restrictions introduced in Sec. II B for the components 1 and 2 the thermal energy vanishes $\epsilon_{T 1}=\epsilon_{T 2}=0$ because these components represent the cold target and projectile components.

In the calculation ${ }^{20}$ the heat conduction vanishes, owing to the symmetry of the discussed problem. So on the basis of Sec. III we use the following expression:

$$
g=\Sigma_{i} \Delta \mu_{i}\left[5 \epsilon_{T i} / 3+n_{i} m \Delta u_{i}^{2} / 2\right]+q_{\text {cond }} .
$$

In the following one dimensional test calculation the interaction energy density $\epsilon_{\text {int }}$ and the interaction term of the pressure tensor $P_{\text {int }}^{i k}$ are calculated as in Sec. III:

$$
\begin{aligned}
& \epsilon_{\text {int }}=\Sigma_{i} n_{i} m \Delta u_{i}^{2} / 2, \\
& P_{\text {int }}^{z z}=2 \epsilon_{\text {int }} .
\end{aligned}
$$

The viscous and heat conductive terms in Eqs. (33) and (35), $P_{\text {visc }}$ and $q_{\text {cond }}$, are given in Sec. IV B.

\section{B. Inclusion of viscous and heat conductive processes in a first order approximation}

In the previous sections the source terms were evaluated only in the zeroth order approximation ${ }^{18}$ for a three component fluid. In the first order approximation we can introduce two new physical properties not discussed yet. By adding a small perturbation to the distribution $f(v)$ we obtain the conventional viscous and heat conductive terms which are driving the system towards a spatial equilibrium. We add these terms to the ones obtained so far:

$$
\begin{aligned}
& \mathscr{g}_{\text {cond }}=\kappa \nabla\left(\epsilon_{T} / n\right) \frac{2}{3}, \\
& P_{\text {visc }}^{j k}=-2 \eta \Lambda^{j k}-(\xi-2 \eta / 3)(\nabla \cdot \mu) \delta^{j k} .
\end{aligned}
$$

These modifications are not strictly derived from a more fundamental theory, they follow only from the assumed similarity between the one and three fluid dynamics.

Here the viscous pressure tensor is just the standard Newtonian one involving the shear viscosity $\eta(\rho, T)$ and the bulk viscosity $\xi(\rho, T)$. Experimentally, very little is known about these coefficients, except for some information from low-energy collective motion such as fission. ${ }^{23,24}$ In principle, one might even question the validity of the Newtonian form for the viscous stress tensor in Eq. (37). Calculations for an ideal Fermi gas tend to show a strong temperature and density dependence according to Refs. 2 and 3

$$
\eta=\sqrt{\pi m\left(T+T_{0}\right)} / 8 \sigma_{\text {tot }},
$$

where $T_{0}$ is the effective temperature of the cold nucleon gas calculated from the average velocity of the degenerate Fermi gas

$$
T_{0}=9\left(3 \pi^{2} / 2\right)^{2 / 3} n^{2 / 3}(\hbar c)^{2} / 16 m c^{2},
$$

and $T$ is evaluated from the total thermal energy density as $T=2 \epsilon_{T} / 3 n$. Since these calculations were not yet verified experimentally for heavy ions 
at high energies, it appears more reasonable to regard $\eta$ as a free parameter and carry out systematic studies regarding its possible influence on the reaction.

\section{Inclusion of the Yukawa and Coulomb potentials}

Under the physical assumptions discussed above, the set of equations governing the heavy-ion collision are the classical equations of hydrodynamics, formulated as conservation equations for mass, momentum, and energy. In the presence of the long range interactions described by interaction potentials the momentum equation reads as ${ }^{25}$

$$
\left[\partial_{t}+(\nabla \cdot u)\right](\rho \mu)=-(\nabla \cdot P)-n \nabla V,
$$

where the symbol $V$ denotes the interaction potentials that were not included in the equation of state of nuclear matter because of their long-range properties. A detailed discussion of how this separation of nucleon-nucleon interactions, into a short-range part incorporated in the equation of state and a long range part treated explicitly, can be justified is given in Ref. 26. $V$ is defined as a sum of a Yukawa and a Coulomb contribution, $V^{y}$ and $V^{C}$. The Yukawa potential is determined from

$$
\left(\nabla^{2}-\alpha^{2}\right) V^{y}(\mu)=-4 \pi \beta n(\mu)
$$

with $\beta=-280 \mathrm{MeV} \mathrm{fm}$ and $\alpha=2.1 \mathrm{fm}^{-1}$. (These values were adjusted to reproduce reasonable surface properties for finite nuclei. ${ }^{26}$ ) For the Coulomb potential a constant charge-to-mass ratio is assumed:

$$
\nabla^{2} V^{C}(\mu)=-4 \pi(\mathrm{Ze} / A)^{2} n(\mu) .
$$

In relativistic calculations the means of introducing potentials is unclear. Therefore up to now no relativistic calculations have incorporated potentials and one has to be content with not being able to describe droplet formation properly. In the Los Alamos calculations, e.g., negative pressures were cut off, because they led to the formation of droplets with unphysical properties. ${ }^{27}$

The equation for the internal energy density does not contain the terms arising from this long range interaction. Moreover, the compressional energy density and the compressional pressure can be eliminated from the equation as in the one fluid models. $^{3,26}$ Thus the whole system of equations we solve is the following:

$$
\begin{aligned}
& {\left[\partial_{t}+(\nabla \cdot u)\right] n=0,} \\
& {\left[\partial_{t}+(\nabla \cdot u)\right](\rho u)=-(\nabla \cdot P)-n \nabla V,} \\
& {\left[\partial_{t}+(\nabla \cdot u)\right] \epsilon_{\mathrm{res}}=-(\nabla \cdot g)-\left(\Lambda: P_{\mathrm{res}}\right),} \\
& {\left[\partial_{t}+(\nabla \cdot u)\right] n_{i}=-\left(\Delta \mu_{i} \cdot \nabla\right) n_{i}+n_{i}(\nabla \cdot u)} \\
& -\Sigma_{j \neq i} Z_{i j}, \quad(i=1,2) .
\end{aligned}
$$

\section{DISCUSSION OF THE MODEL AND ITS CONSEQUENCES}

\section{A. Results of one dimensional calculations}

The model described above is solved numerically with a Eulerian method ${ }^{28}$ in one dimension. The equation of state was that of an ideal Boltzmann gas mixture with an additive compressional term taken from Ref. 29. From the long range potentials only the Yukawa potential was taken into account and in the present calculation we neglected the temperature dependence of the viscosity $(\eta=20$ $\left.\mathrm{MeV} / \mathrm{fm}^{2} c, \xi=0, \kappa=0.015 \mathrm{c} / \mathrm{fm}\right)$. The schedule of the solution was as follows: From Eqs. (43) we obtained $n, n_{1}, n_{2}, u$, and $\epsilon_{\text {res }}$ in each integration step. Since the velocities of components 1 and 2 are fixed: $\Delta u_{1}=u_{1}-u, \Delta u_{2}=u_{2}-u$, so from Eq. (14) $n_{3}$ and $u_{3}$ can be obtained. Using the expression for the energy density [Eqs. (33) and (34)] the temperature $T_{3}$ can be evaluated, as can the source terms [right hand sides of Eqs. (43)] for the following step. The details of the solution method are equivalent to the ones of Ref. 3.

A symmetric system was analyzed corresponding to a central (slab) heavy-ion collision of 200 $\mathrm{MeV} /$ nucleon projectile energy. The nucleonnucleon cross section determining the collision number $Z$ was varied and the penetration length of components 1 and 2 was studied (Figs. $2-4$ ). The thermalized component developed rapidly for realistic cross sections and the interpenetration of components 1 and 2 was relatively small.

In Fig. 2 the results of a calculation with realistic cross section $\sigma=25 \mathrm{mb}$ are shown. At the compression phase [Fig. 2(a)] the interpenetration of components 1 and 3 is about $3 \mathrm{fm}$ although the observable width of the shock front is only $1.5 \mathrm{fm}$. The velocity of the 3rd component in the shock front drops down to zero faster than that of the whole matter, because components 1 and 2 maintain their original average velocities and their momentum is balanced by the 3 rd component. At the stage of maximum compression [Fig. 2(b)] there remains 

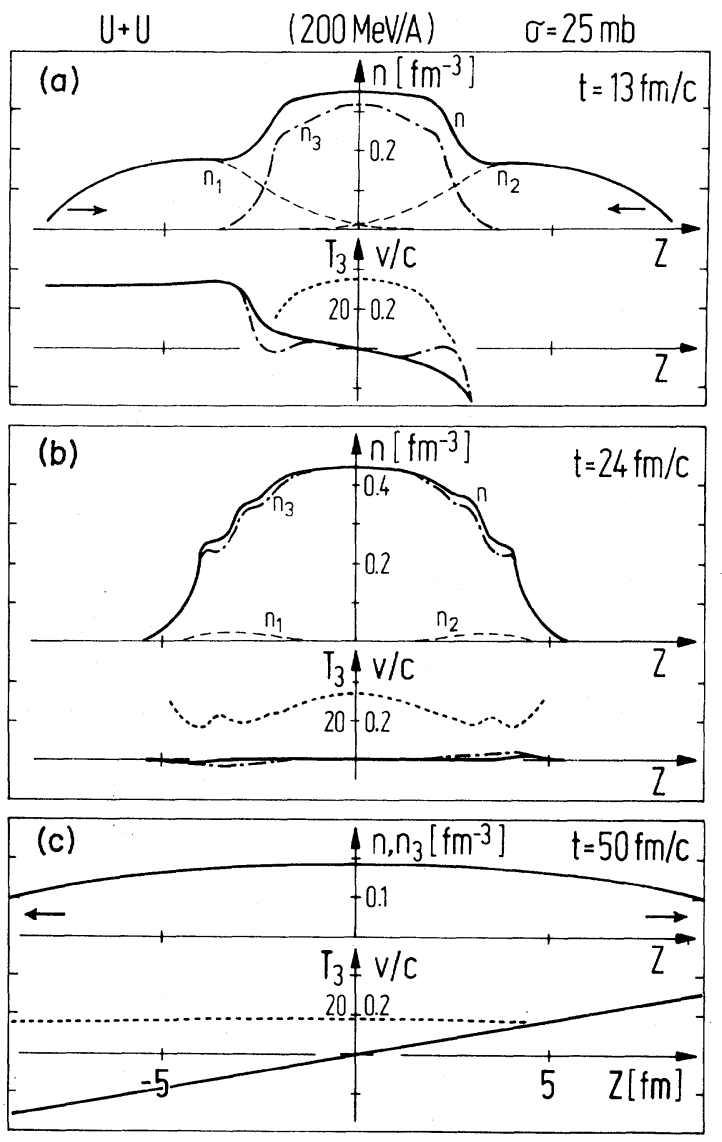

FIG. 2. The density distributions of the different components and their time development in a one dimensional calculation describing a $\mathrm{U}+\mathrm{U}$ collision at 200 $\mathrm{MeV} /$ nucleon bombarding energy. The nucleon-nucleon cross section is $\sigma=25 \mathrm{mb}$. Full lines belong to the whole matter density and velocity distributions, dashed lines to the partial densities of components 1 and 2, the dasheddotted lines indicate the partial density and velocity of the 3rd thermal component, and the dotted lines show its temperature $T_{3}$ in units of $\mathrm{MeV}$. The three sets of figures (a), (b), and (c) belong to the indicated times. The overlap of components 1 and 3 is $\simeq 3 \mathrm{fm}$ at the initial phase.

only a very small fraction of the cold components, while at late expansion stages [Fig. 2(c)] we already have a fully thermalized matter which expands with a velocity increasing linearly outwards.

In this model it is possible to follow the transition from the two independent interpenetrating fluids towards the conventional one fluid description. If we increase the cross section to infinity component 1 or 2 vanishes immediately in the presence of any other component and consequently we have a sharp surface between the thermalized and
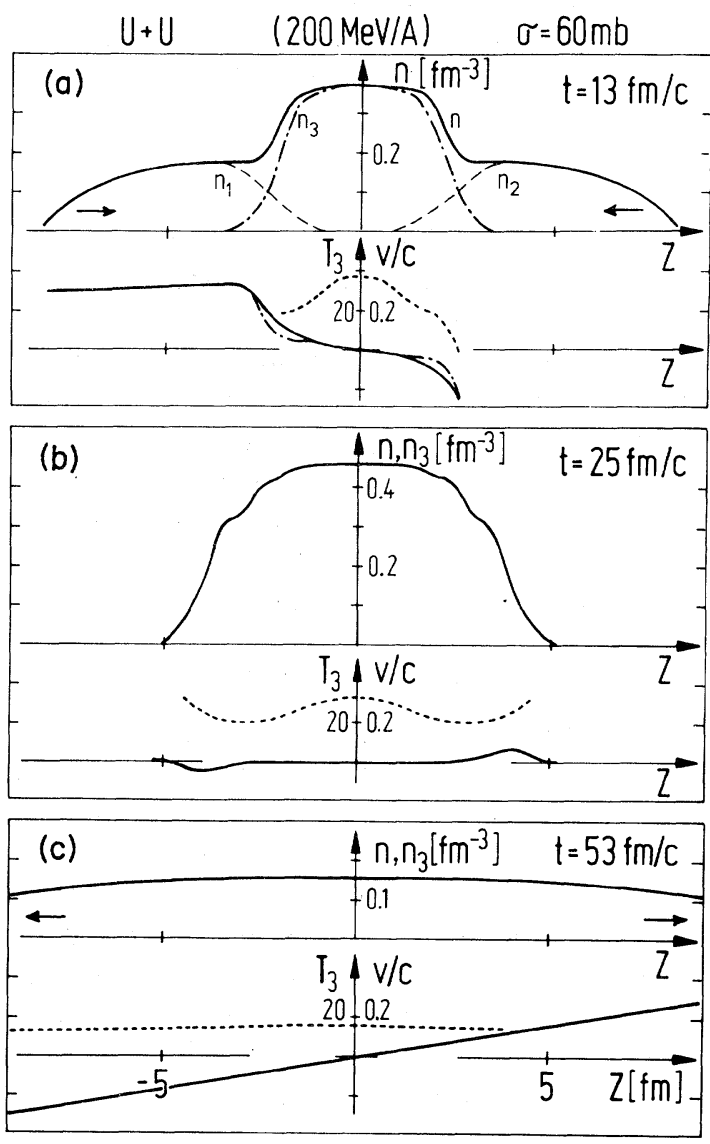

FIG. 3. The same as Fig. 2 with $\sigma=60 \mathrm{mb}$.

cold components. Thus, to each space time point a unique velocity, temperature, and density can be attributed like in the one fluid case. This is obvious if we consider Eqs. (17) or (43). In these equations the second equation describes the transition from the cold components to the thermalized one. In Fig. 3 the results of a calculation are shown with increased cross section $\sigma=60 \mathrm{mb}$. In the compression the interpenetration is reduced to $2 \mathrm{fm}$ and at the full compression we already have no cold components.

On the other hand, if we assume that the cross section goes to zero we reach the noninteracting limit of the two-fluid model. Since we have seen that the separated Eqs. (8) are mathematically equivalent to Eqs. (17) we expect that the latter equations also reproduce the two fluid properties. For a perfect fluid this can be seen analytically but a problem arises when we want to introduce generalizations (see Sec. IV) which reflect the properties of the realistic nuclear matter (potentials, viscosity, heat conduction, compressional energy, etc.). Since the separation of these processes into 

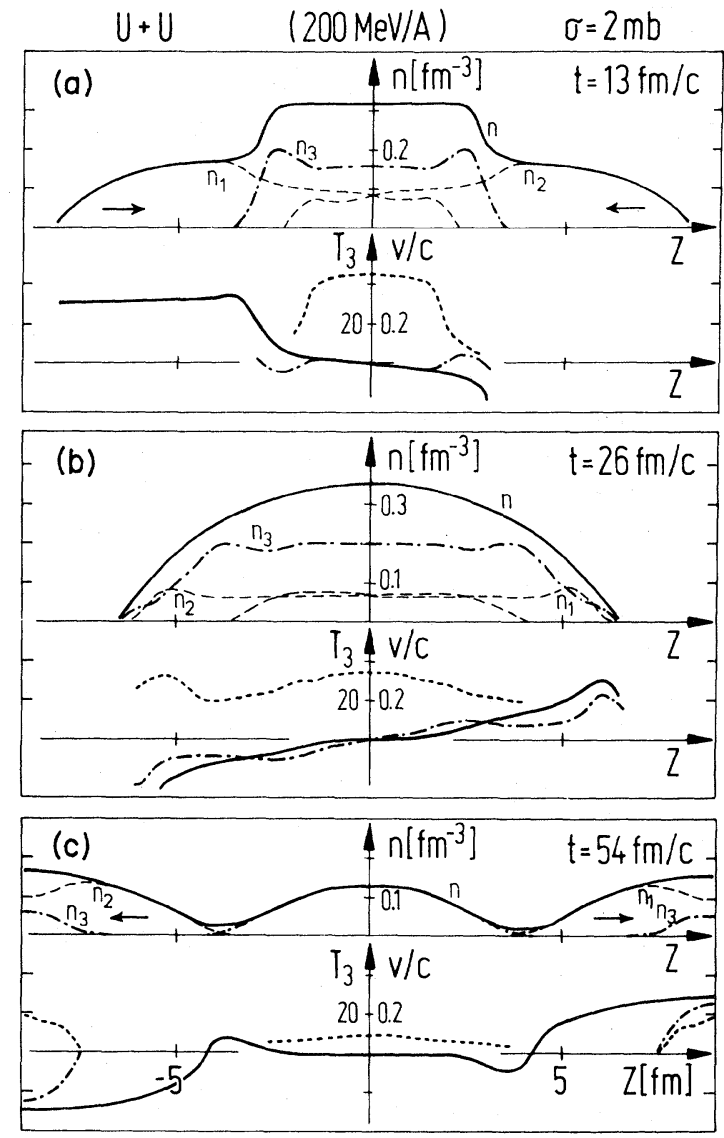

FIG. 4. The same as Fig. 2 with $\sigma=2 \mathrm{mb}$.

components is by no means trivial, their inclusion in the separated version of the model [Eqs. (8) or (12)] needs further considerations. To analyze the decoupling of the components from each other a calculation was performed using Eqs. (43) with a strongly decreased cross section $\sigma=2 \mathrm{mb}$ (Fig. 4). Here components 1 and 2 survive the collision and leave the system with a density and velocity close to the initial ones. It is interesting to note that a sizable amount of the 3rd component remains in the middle of the reaction zone and the cold matter moving outwards drags some thermalized component with itself. Hence, the nuclei interpenetrating each other obtain an observable thermal excitation.

\section{B. Evolution of the momentum distribution}

The momentum distribution in the central region changes rapidly in time and can be compared to the calculations of Ref. 12 made in the framework of the kinetic transport theory. In Fig. 5 the time evolution of the velocity distributions are plotted for a central region of length $\Delta z$

$$
\left.f(v, t)\right|_{\Delta z}=\int_{-\Delta z / 2}^{\Delta z / 2} f(z, v, t) d z,
$$

where $f(z, v, t)$ is the distribution given by Eqs. (13) and (18). The similarity suggests that although our model simplifies the kinetic aspects, the basic features of the evolution of momentum distribution are reflected properly. The velocity distribution

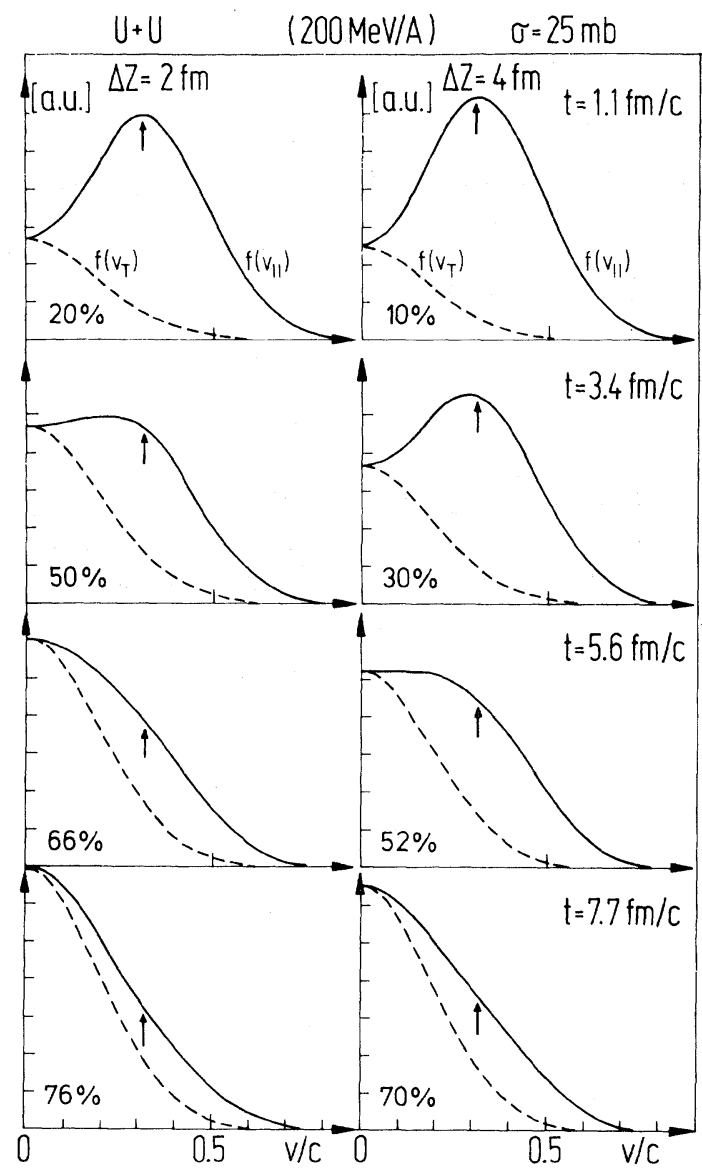

FIG. 5. Time development of the momentum distribution of the central region in the beam direction and orthogonally to it. The speed of thermalization is similar to the one obtained in the cascade and kinetic models. The arrows indicate the velocity of the projectile/target in c.m. and the given percent values show the degree of the thermalization $\left\langle n_{3}\right\rangle /\langle n\rangle$ in the spatial region $\Delta z$. The local thermalization is somewhat stronger than in kinetic models because of the strong compression in the interaction region; however, if we take longer spatial intervals $(\Delta z=4 \mathrm{fm}$ or more) the average thermalization speed decreases because of the gradual interpenetration. 
reaches an almost complete thermalization at the stage of full compression in a central $\mathrm{U}+\mathrm{U}$ collision at $200 \mathrm{MeV} /$ nucleon energy $[t=24 \mathrm{fm} / c$, Fig. 2(b)]. Before this stage the degree of thermalization (ratio of the 3rd component to the whole matter) is different in different locations. Already at an intermediate stage $[t=13 \mathrm{fm} / c$, Fig. 2(a) $]$ the matter is thermalized to $90 \%$ in the middle but at the same moment the matter in the compressed zone $(-3 \mathrm{fm}<z<3 \mathrm{fm}$ ) is thermalized to $50 \%$ only. Thus, the process of thermalization is by no means a minor effect during the collision.

Finally, in Fig. 6 we show the qualitative difference between the velocity distributions of the twofluid model and ours. While in the two-fluid model [Fig. 6(b)] the average velocities of the two fluids approach each other owing to the drag terms in the Euler equation, in our model [Fig. 6(a)] a third thermal component develops and the resulting total velocity distribution may approach a thermalized one more smoothly.

\section{Conclusions}

At the end we answer qualitatively the question: What are the observable physical consequences of the explicit consideration of the thermalization process in a fluid dynamical model?

By comparing the calculations with large and
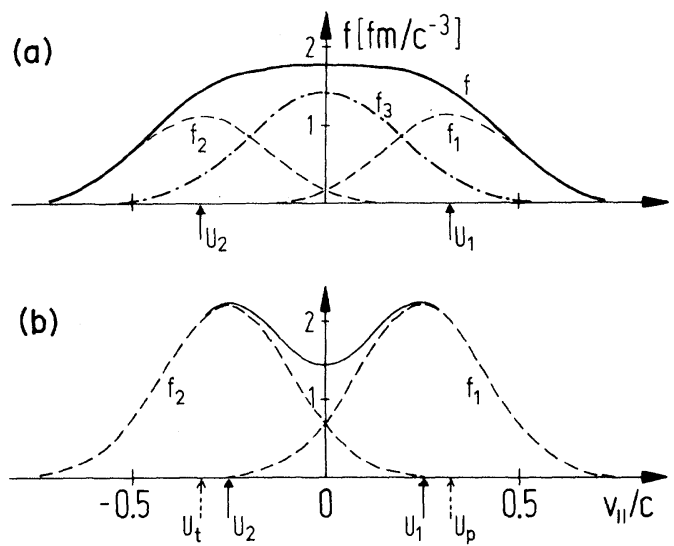

FIG. 6. Velocity distribution in the beam direction calculated in the three-component fluid dynamical model (a) in the middle of the reaction zone $(z=0)$ for the case shown in Fig. 2(a). (b) Schematic plot of the velocity distribution in a two fluid model like in Ref. 8. The two peaks are approaching each other due to the interaction between the two fluids. small cross sections we observe an important difference: In the small cross section case the maximum compression and thermal excitation is essentially decreased. This is the consequence of the fact that in this case the existing cold components maintain their kinetic energy and so the compressional energy is smaller. The density increase is also smaller and the momentum of the incoming cold matter is balanced by the large interaction pressure $p_{\text {int }}^{z z}=2 \epsilon_{\text {int }}$. However, the interaction pressure in the transverse directions vanishes in the ideal gas approximation and this means that all the compressional, thermal, and interaction pressure components Eq. (33) are smaller in transverse directions. Consequently the presence of the cold matter components decreases the momentum transfer into transverse directions.

This effect may weaken somewhat the "sidesplash" process and may change the deflection function $\theta(b)$ of the "bounce-off" 30 effect. The deflection angle $\theta$ will be slightly smaller especially at large impact parameters where the possibility of interpenetration is larger.

We have seen, however, that in the examples presented above (Fig. 2) the thermalization is nearly complete. This result shows that the threecomponent fluid model yields similar results to the one fluid one in the case of central and nearly central collisions in the some $100 \mathrm{MeV} /$ nucleon bombarding energy region. Thus the strong hydrodynamical effects-the bounce of ${ }^{30}$ observed in the sideward peaked angular $p, d$, and $t$ cross sections, ${ }^{31}$ in two-proton correlation experiments with heavy targets, ${ }^{32,33}$ and also in correlations between light and heavy ejected fragments ${ }^{34}$-which are caused mainly by central collisions will not be essentially changed in the three-component fluid dynamical model.

In the three dimensional version of the model the inclusive spectra are expected to be reproduced better than in the one and two fluid models and it should provide us with a reliable description of collisions in the few $\mathrm{GeV}$ per nucleon energy region as well.

\section{ACKNOWLEDGMENTS}

Enlightening discussions with J. Bondorf and P. Danielewicz are gratefully acknowledged. This work was supported by the Bundesministerium für Forschung und Technologie, by the Gesellschaft für Schwerionenforschung, and by the Alexander von Humboldt Stiftung. L. P. C. is a fellow of the Alexander von Humboldt Foundation. 
1P. J. Siemens and J. I. Kapusta, Phys. Rev. Lett. $\underline{43}$, 1486 (1979).

${ }^{2}$ L. P. Csernai, B. Lukács, and J. Zimányi, Lett. Nuovo Cimento 27, 111 (1980); L. P. Csernai and H. W. Barz, Z. Phys. A 296, 173 (1980).

${ }^{3}$ G. Buchwald, L. P. Csernai, J. Maruhn, W. Greiner, and H. Stöcker, Phys. Rev. C 24, 135 (1981).

${ }^{4}$ J. R. Nix, Prog. Part. Nucl. Phys. 2, 237 (1979).

5J. Knoll, Phys. Rev. C 20, 773 (1979); J. Knoll and J. Randrup, Nucl. Phys. A324, 445 (1979); J. Knoll, ibid. A343, 511 (1980).

6J. Cugnon, T. Mizutani, and J. Vermeulen, Nucl. Phys. A352, 505 (1981); J. Cugnon and S. E. Koonin, ibid. A355, 477 (1981); J. Cugnon, J. Knoll, and J. Randrup, ibid. A 360,444 (1981).

${ }^{7}$ J. P. Bondorf, Yu. B. Ivanov, and J. Zimányi, Phys. Scr. 24, 514 (1981).

${ }^{8}$ A. A. Amsden, A. S. Goldhaber, F. H. Harlow, and J. R. Nix, Phys. Rev. C 17, 2080 (1978).

${ }^{9}$ M. Gyulassy and W. Greiner, Ann. Phys. (N.Y.) 109, 485 (1977).

10I. Montvay and J. Zimányi, Nucl. Phys. $\underline{\text { A316, }} 490$ (1979).

${ }^{11} \mathrm{~L}$. D. McLerran, in Proceedings of the International Workshop on Gross Properties of Nuclei and Nuclear Excitations, Hirschegg, 1981, edited by H. Feldmeier (Technische Hochscule, Darmstadt, 1981), p. 9.

12J. Randrup, Nucl. Phys. A314, 429 (1979).

${ }^{13}$ H. J. Pirner and B. Schürmann, Nucl. Phys. A316, 461 (1979).

${ }^{14} \mathrm{P}$. Danielewicz, in Proceedings of the International Conference on Extreme States in Nuclear Systems, Contributed Papers, 1980, Dresden, Zentralinstitut für Kernforschung Dresden Report No. 404, 1980, p. 10.

${ }^{15}$ K. K. Gudima, H. Iwe, and V. D. Toneev, J. Phys. G 5, 229 (1979).

${ }^{16}$ Y. Yariv and Z. Fraenkel, Phys. Rev. C 20, 2227 (1979).

${ }^{17}$ R. Malfliet, Phys. Rev. Lett. 44, 864 (1980); Nucl. Phys. A363, 429 (1981); 456 (1981).

${ }^{18}$ K. Huang, Statistical Mechanics (Wiley, New York, 1963).

${ }^{19}$ W. B. Thompson, An Introduction to Plasma Physics (Pergamon, New York, 1962).

${ }^{20}$ I. Lovas, Nucl. Phys. A 367, 509 (1981).

${ }^{21}$ K. H. Müller and S. Bohrmann, Lawrence Berkeley Laboratory Report No. LBL-12663, 1981 (unpublish- ed).

${ }^{22}$ A. Rosenhauer, L. P. Csernai, J. Maruhn, and W. Greiner, University of Frankfurt Report No. UFTP65, 1981 (unpublished).

${ }^{23}$ J. R. Nix and A. P. Sierk, Los Alamos Scientific Laboratory Report No. LA-UR-79-1623, 1979.

${ }^{24}$ R. Wieczorek, R. W. Hasse, and G. Süssmann, Physics and Chemistry of Fission (IAEA, Vienna, 1974), Vol. I, p. 523.

25J. A. Maruhn and W. Greiner, in Heavy Ion Science, edited by A. Bromley (Plenum, New York, 1982), Vol. I.

${ }^{26}$ C. Y. Wong, J. A. Maruhn, and T. M. Welton, Nucl. Phys. A253, 469 (1975); Phys. Lett. 66B, 19 (1977).

${ }^{27}$ A. A. Amsden, F. H. Harlow, and J. R. Nix, Phys. Rev. C 15, 2059 (1977).

28J. P. Boris and D. L. Book, J. Comp. Phys. 11, 38 (1973).

${ }^{29}$ J. A. Maruhn, thesis, University of Frankfurt (1976); K. A. Bruckner, J. R. Buchler, R. C. Clark, and R. J. Lombard, Phys. Rev. 181, 1543 (1969); H. A. Bethe, Annu. Rev. Nucl. Sci. 21, 93 (1971).

${ }^{30}$ H. Stöcker, J. Maruhn, and W. Greiner, Z. Phys. A 290, 297 (1979); 293, 173 (1979); Phys. Rev. Lett. 44, 725 (1980); H. Stöcker, L. P. Csernai, G. Graebner, G. Buchwald, H. Kruse, R. Y. Cusson, J. A. Maruhn, and W. Greiner, Phys. Rev. C 25, 1873 (1982).

${ }^{31}$ H. H. Gutbrod, Lawrence Berkeley Laboratory Report LBL-11123, 1980; in Proceedings of the Symposium on High Energy Nuclear Interactions, Hakone, Japan, 1980, edited by K. Nakai and A. S. Goldhaber, p. 93; R. Stock, H. H. Gutbrod, W. G. Meyer, A. M. Poskanzer, A. Sandoval, J. Gosset, C. H. King, G. King, Ch. Lukner, Nguyen Van Sen, G. D. Westfall, and K. L. Wolf, Phys. Rev. Lett. 44, 1243 (1980).

${ }^{32}$ I. Tanihata, M.-C. Lemaire, S. Nagamiya, and S. Schnetzer, Phys. Lett. 97B, 363 (1980); S. Nagamiya, L. A. Anderson, W. Bruckner, O. Chamberlain, M. C. Lemaire, S. Schnetzer, G. Shapiro, H. Steiner, and I. Tanihata, Phys. Lett. $\underline{81 B}, 174$ (1979).

${ }^{33}$ L. P. Csernai and W. Greiner, Phys. Lett. 99B, 85 (1981); L. P. Csernai, W. Greiner, H. Stöcker, I. Tanihata, S. Nagamiya, and J. Knoll, Phys. Rev. C (to be published).

${ }^{34}$ W. G. Meyer, H. H. Gutbrod, Ch. Lukner, and A. Sandoval, Phys. Rev. C 22 179 (1980). 